\title{
ON THE ZEROS OF SECOND ORDER LINEAR DIFFERENTIAL POLYNOMIALS
}

\author{
by J. K. LANGLEY
}

(Received 9th January 1989)

\begin{abstract}
We determine all functions $f(z)$ meromorphic in the plane such that $f^{\prime}(z) / f(z)$ has finite order and $f(z)$ and $F(z)$ have only finitely many zeros, where $F(z)=f^{\prime \prime}(z)+A f(z)$ for some constant $A$.
\end{abstract}

1980 Mathematics subject classification (1985 Revision): 30D35.

\section{Introduction}

Our starting point is the following result of Frank, Hennekemper and Polloczek [3]:

Theorem A Suppose that $f(z)$ is meromorphic in the plane, and that $f(z)$ and $f^{(k)}(z)$ have only finitely many zeros, for some $k \geqq 3$. Then $f^{\prime}(z) / f(z)$ is rational, that is $f(z)=R(z) \exp (P(z))$, where $R(z)$ is rational and $P(z)$ is a polynomial.

A comparable result classifying functions $f(z)$ meromorphic in the plane such that $f(z)$ and $f^{\prime \prime}(z)$ have only finitely many zeros is not known. It is natural to extend the above problem to consideration of the zeros of a meromorphic function $f(z)$ and a linear differential polynomial $F$ in $f(z)$, that is

$$
F(z)=f^{(k)}(z)+\sum_{j=0}^{k-1} a_{j}(z) f^{(j)}(z)
$$

where the $a_{j}$ are, say, rational. Among other results in [2], Frank and Hellerstein classified completely those entire functions $f(z)$ such that $f(z)$ and $F(z)$ have only finitely many zeros, where $F$ is given by (1.1) with $k \geqq 2$ and the $a_{j}$ polynomials. They also showed that if $f$ is meromorphic in the plane, and $f$ and $F$ have only finitely many zeros, where $k \geqq 3$ and the $a_{j}$ are again polynomials, then $f^{\prime} / f$ has finite order determined by the degrees of the $a_{j}$. For constant coefficients, Steinmetz proved the following [15]:

Theorem B. Suppose that $f(z)$ is meromorphic and non-constant in the plane and that $f(z)$ and $F(z)$ have no zeros, where $F(z)$ is given by (1.1) with $k \geqq 3$ and $a_{0}, \ldots, a_{k-1}$ constants. Then $f$ satisfies one of the following: 


$$
\begin{gathered}
f(z)=\exp \left(a z+b+e^{c z+d}\right) \\
f(z)=e^{a z+b}\left(e^{c z+d}-1\right)^{-n} \\
f(z)=e^{a z+b}(z-c)^{-n}
\end{gathered}
$$

Here $a, b, c, d$ are constants and $n$ is a positive integer.

The following was proved in [12];

Theorem C. Suppose that $f(z)$ is meromorphic of finite order in the plane, and that $f(z)$ and $F(z)$ have only finitely many zeros, where $F(z)=f^{\prime \prime}(z)-\alpha f(z)$ for some constant $\alpha$. If $\alpha=0$, then $f^{\prime} / f$ is rational. If $\alpha \neq 0$, then either $f^{\prime} / f$ is rational, or $f$ is given by (1.3).

The case $\alpha=0$ in Theorem $C$ represents a slight improvement of a result of Mues [14]. Now the hypothesis that $f$ has finite order appears in Theorem $C$ because the method of [12] uses asymptotic integration in sectors for $f$, with the PhragmenLindelöf principle used to "fill in the gaps". With a weaker assumption on $f$ we shall prove here:

Theorem 1. Suppose that $f(z)$ is meromorphic in $|z| \geqq R$, and that $a_{0}(z)$ and $a_{1}(z)$ are analytic there, with

$$
a_{j}(z)=0\left(|z|^{j-2}\right)
$$

Suppose that $f(z) F(z)$ has no zeros in $|z| \geqq R$, where $F$ is given by

$$
F(z)=f^{\prime \prime}(z)+a_{1}(z) f^{\prime}(z)+a_{0}(z) f(z)
$$

Suppose finally that $\bar{N}(r, f)$ has finite lower order. Then $f^{\prime} / f$ has only finitely many poles in $|z| \geqq R$, with a pole or removable singularity at infinity.

Here $\bar{N}(r, f)$ counts the points at which $f$ has poles, each counted just once (see Section 2).

Corollary. If $f(z)$ is meromorphic in the plane, if $f(z)$ and $f^{\prime \prime}(z)$ have only finitely many zeros, and if $f^{\prime} / f$ has finite order, then $f^{\prime} / f$ is rational.

Theorem 1 is sharp at least to the extent that the coefficients $a_{j}(z)$ cannot be made any larger. For example, $f(z)=\sec (\sqrt{ } z)$ has no zeros, and nor has

$$
F(z)=f^{\prime \prime}(z)+(1 / 2 z) f^{\prime}(z)+(1 / 4 z) f(z)=f^{3}(z) / 2 z
$$


It seems reasonable to believe that Theorem 1 would be true without any restriction on $\bar{N}(r, f)$. A comparable result is proved in [13] for $k$ at least 3 and small rational coefficients in (1.1). Also [11] contains a result with extra hypotheses on $f$ and $F$, but which allows slightly larger coefficients $a_{j}$. The assumption made on $\bar{N}(r, f)$ in Theorem 1 is however considerably weaker than that of Theorem $\mathrm{C}$, making no restriction on the multiplicities of poles of $f$.

For the case $\alpha \neq 0$ of Theorem $\mathrm{C}$, we have the following:

Theorem 2 Suppose that $f(z)$ is meromorphic in the plane, and that $f(z)$ and $F(z)$ have only finitely many zeros, where $F(z)=f^{\prime \prime}(z)-\alpha f(z)$ for some non-zero constant $\alpha$. If $\bar{N}(r, f)$ has finite lower order, then either $f^{\prime} / f$ is rational, or $f$ is given by (1.2) or (1.3).

We make the following remark about the proof of Theorems 1 and 2 above. Suppose for example that $f$ and $f^{\prime \prime}$ have only finitely many zeros. Then, as in [14], the function $z-\left(f / f^{\prime}\right)$ is a yuotient of solutions of an equation $w^{\prime \prime}+b w=0$, where $b$ has only finitely many poles. With our assumptions, $b$ turns out to be rational. The proof then depends on demonstrating the existence of unbounded regions where $f^{\prime} / f$ has infinitely many poles whose residues are incompatible with arising from poles of $f$. This is the key to both proofs. It seems very difficult however to apply this in the general case where $b$ may be transcendental.

\section{Preliminaries}

We use the following notation. For $r \geqq 0$, and $\alpha, \beta$ real, then

$$
S(r, \alpha, \beta)=\{z:|z|>r, \alpha<\arg z<\beta\} .
$$

We use $W(u, v, w)$ to denote the Wronskian of $u, v, w$.

We need the Nevanlinna theory for functions meromorphic in $0<R \leqq|z|<+\infty$. (See for example [1, p. 98].) For such a function $f(z)$, we have a representation $f(z)=$ $z^{n} h(z) G(z)$, where $n$ is an integer, $h(z)$ is analytic in $|z| \geqq R$ with a removable singularity at infinity, and $G(z)$ is meromorphic in the plane. (See $[16$, p. 15] for a proof of this fact.) We can thus define Nevanlinna functionals $m(r, f), N(r, f)$, etc. for $r \geqq R$, where

$$
N(r, f)=\int_{R}^{r} n(t, f) d t / t
$$

and $n(t, f)$ is the number of poles of $f$, counting multiplicities, in $R \leqq|z| \leqq t . \bar{N}(r, f)$ is defined similarly, with multiple poles counted just once. The first fundamental theorem, in this setting, becomes, for finite $a$,

$$
T(r, 1 /(f-a))=T(r, f)+O(\log r)
$$

Now $f$ has a pole or removable singularity at infinity if and only if $T(r, f)=O(\log r)$ 
through a sequence tending to infinity. Moreover, $T(r, f)$ differs from a non-decreasing function by a term which is $O(\log r)$. Denoting by $S(r, f)$ any quantity which is $O\left(\log ^{+} T(r, f)+\log r\right)$, possibly outside a set of finite linear measure, we have $m\left(r, f^{\prime} / f\right)=S(r, f)$. We can define the order and lower order of such terms just as in $\left[6\right.$, p. 16]. Finally we remark that Clunie's lemma is valid in this context. If $P(f) f^{n}=Q(f)$, where $P(f)$ and $Q(f)$ are differential polynomials in $f$ whose coefficients $a(z)$ satisfy $m(r, a)=S(r, f)$, and if $Q(f)$ has total degree at most $n$ in $f$ and its derivatives, then $m(r, P(f))=S(r, f)$. The proof is identical to that in $[6$, p. 68].

\section{Lemmas needed for the proofs of Theorems 1 and 2}

Our proofs make extensive use of Hille's method of asymptotic integration of the equation

$$
y^{\prime \prime}+a_{1} y^{\prime}+a_{0} y=0
$$

(See [8, Chapter 7], or [9]). Suppose that $a_{1}$ and $a_{0}$ are analytic in a sector $S_{1}=S\left(r_{1}, \theta_{0}-\alpha, \theta_{0}+\alpha\right)$, such that $a_{1}(z)=0\left(|z|^{-1}\right)$ and $a_{1}^{\prime}(z)=0\left(|z|^{-2}\right)$ and

$$
a_{0}(z)=\gamma z^{n}(1+0(1 /|z|))
$$

there, where $n \geqq-1, \gamma$ is a non-zero constant and $0 \leqq \alpha \leqq 2 \pi /(n+2)$, and $\alpha \leqq \pi$ if $n=-1$. We first make a change of variable $y=u v$, where $v^{\prime} / v=-a_{1} / 2$, so that $u$ satisfies

$$
u^{\prime \prime}+b(z) u=0
$$

and $b(z)=\gamma z^{n}(1+o(1))$ as $z$ tends to infinity in $S_{1}$. Now take a large $z_{0}$ in $S_{1}$ : setting

$$
Z=\int_{z_{0}}^{z} b(t)^{1 / 2} d t=\beta z^{(n+2) / 2}(1+o(1))
$$

where $\beta=\gamma^{1 / 2} 2 /(n+2)$, makes $Z$ analytic and one-one in a sector

$$
S_{2}=S\left(r_{2}, \theta_{0}-\alpha+\varepsilon, \theta_{0}+\alpha-\varepsilon\right)
$$

Here $r_{2}$ depends on $\varepsilon$, which may be chosen arbitrarily small and positive. Now (3.1) has linearly independent solutions

$$
v(z) b(z)^{-1 / 4}(1+o(1)) \exp \left((-1)^{k} i Z\right)
$$

in $S_{2}$, for $k=1,2$, and any solution $y$ of (3.1) satisfies

$$
\log ^{+}|y(z)|=0\left(|z|^{(n+2) / 2}\right)
$$


there. If $\arg z=\theta_{0}$ is a critical ray for (3.3), that is

$$
\operatorname{Arg} \gamma+(n+2) \theta_{0}=0(\bmod 2 \pi)
$$

then $\operatorname{Re}\left(i \beta z^{(n+2) / 2}\right)=0$ on this ray, and if $\left|\theta-\theta_{0}\right|$ is small and positive, then for large $z$ on $\arg z=\theta$, one of the solutions (3.5) is large and the other is small. We need:

Lemma 1. Suppose that $a_{1}$ and $a_{0}$ are rational, such that $a_{1}$ vanishes at infinity and $a_{0}$ satisfies (3.2) with $\gamma \neq 0$ and $n \geqq-1$. Suppose further that (3.1) has linearly independent solutions which are meromorphic in the plane. Then $n \neq-1$. Also if $n=0$ then there exist a non-zero constant $A$ and rational functions $R_{1}, R_{2}$ such that $R_{1}(z) e^{A z}$ and $R_{2}(z) e^{-A z}$ are solutions of (3.1).

Proof. The first part is essentially Theorem 2 of [7]. If $n=-1$, we can choose $\theta_{0}$ satisfying (3.7), and for a small positive $\varepsilon$, determine a non-trivial solution $y$ of (3.1) such that for some positive $c_{1}$,

$$
\log |y(z)| \leqq-c_{1}|z|^{1 / 2}
$$

in a sector $S\left(r_{2}, \theta_{0}+\varepsilon, \theta_{0}+2 \pi-\varepsilon\right)$. But $y(z)$ has only finitely many poles and order at most $1 / 2$, and so must be rational, by (3.8) and the Phragmén-Lindelöf principle [4, p. 104]. This is a contradiction, as no rational function can satisfy (3.8) without vanishing identically.

For the second part, we can again take $\theta_{0}$ satisfying (3.7). Now (3.4) becomes

$$
Z=i A z+b_{1} \log z+0(1 /|z|)
$$

for some constants $b_{1}$ and $A$ with $A \neq 0$. Thus (3.1) has a solution satisfying

$$
y(z)=z^{b_{2}}(1+o(1)) e^{A_{1} z}
$$

in $S_{3}=S\left(r_{3}, \theta_{0}+\varepsilon, \theta_{0}+\pi-\varepsilon\right)$, say, with $b_{2}$ a constant and $A_{1}= \pm A$, and such that $\exp \left(A_{1} z\right)$ tends to zero in $S_{3}$. Determining a representation for $y$ in $S_{4}=$ $S\left(r_{3}, \theta_{0}-\pi+\varepsilon, \theta_{0}-\varepsilon\right)$, we see that since $y$ has only finitely many poles and order at most 1 , then $y(z) \exp \left(-A_{1} z\right)$ must be rational, using the Phragmén-Lindelöf principle again. We can make a second such solution similarly.

Lemma 2. Suppose that $f(z)$ is meromorphic in $|z| \geqq R$, and that $f$ and $F$ have no zeros there, where

$$
F(z)=f^{\prime \prime}(z)+a_{1}(z) f^{\prime}(z)+a_{0}(z) f(z)
$$

and $a_{0}, a_{1}$ are analytic in $|z| \geqq R$ and satisfy (1.4). Then for all $r$ outside a set of finite linear measure we have

$$
T\left(r, f^{\prime} / f\right)=0(\bar{N}(r, f)+\log r)
$$


Proof. Obviously we may assume that $H=f^{\prime} / f$ has an essential singularity at infinity, for otherwise there is nothing to prove. We follow the now standard TumuraClunie method [6, pp. 69-73].

Setting $G=F / f$, and $A=H^{\prime}-G^{\prime} H / 2 G$, we obtain $m(r, A)=S(r, H)$ from Clunie's lemma. We define $h$ formally by $h^{2}=G$, so that setting $g=a_{1}+h^{\prime} / h$ we have

$$
G=(H+g / 2)^{2}+B
$$

where $B=A+a_{0}-g^{2} / 4$ satisfies $m(r, B)=S(r, H)$. Writing $U=H+g / 2$, we obtain $U P=C$, where $m(r, C)=S(r, H)$ and $P=2 U^{\prime}-\left(G^{\prime} / G\right) U$. Thus $m(r, P)=S(r, H)$ by Clunie's lemma. If $P$ is not identically zero, we get

$$
m(r, U) \leqq m(r, P U)+m(r, 1 / P) \leqq N(r, P)+S(r, H)
$$

and the conclusion follows. If $P$ vanishes identically, then $G=U^{2}+B=c U^{2}$ for some constant $c$. If $c \neq 1$, then $m(r, U)=S(r, H)$ so that $m(r, H)=S(r, H)$. Finally if $c=1$ then substituting $H=U-g / 2$ into $G=H^{2}+H^{\prime}+a_{1} H+a_{0}$ and using the fact that $h^{\prime} / h=U^{\prime} / U$ we obtain

$$
g^{2} / 4-g^{\prime} / 2-g a_{1} / 2+a_{0}=0
$$

which implies that $g$ is analytic in $|z|>R$ and has a removable singularity at infinity, with $g(\infty)=0$. Hence $G$ is analytic in $|z|>R$ with at most a pole at infinity, and the same is true for $H$, by Clunie's lemma.

\section{Proof of Theorem 1}

We assume that there exists a function $f(z)$ meromorphic in $|z| \geqq R>0$ such that $f F$ has no zeros there, where

$$
F(z)=f^{\prime \prime}(z)+a_{1}(z) f^{\prime}(z)+a_{0}(z) f(z)
$$

and $a_{1}(z), a_{0}(z)$ are analytic in $|z| \geqq R$ with

$$
a_{j}(z)=O\left(|z|^{j-2}\right)
$$

We assume that $\bar{N}(r, f)$ has finite lower order, and will show that $f^{\prime} / f$ has only finitely many poles in $|z| \geqq R$, with at most a pole at infinity.

We take a sector $S_{0}=S(R, \alpha, \alpha+2 \pi)$ for some real $\alpha$, and define a function $g$ analytic on $S_{0}$ by

$$
g^{2}=f / F
$$


We remark that $g$ might not be meromorphic on $|z| \geqq R$, but certainly $g^{2}$ is, and so is $g^{\prime} / g$.

Now the equation

$$
y^{\prime \prime}+a_{1} y^{\prime}+a_{\mathrm{o}} y=0
$$

has by (4.2) a regular singular point at infinity, so that by [10, Chapter 15], (4.4) has a solution $f_{1}(z)$ analytic in $S_{0}$ such that for some constant $\gamma_{1}$, the function $h_{1}(z)=$ $f_{1}(z) z^{-\gamma_{1}}$ has a removable singularity at infinity, with $h_{1}(\infty)=1$. We can also define a function $W$ analytic in $S_{0}$ by

$$
W^{\prime} / W=-a_{1}
$$

so that for some constant $\gamma_{2}$, the function $h_{2}(z)=W(z) z^{-\gamma_{2}}$ is again analytic in $|z| \geqq R$, with a removable singularity at infinity, and with $h_{2}(\infty)=1$. Finally we can define a second solution $f_{2}$ of $(4.4)$ by

$$
W\left(f_{1}, f_{2}\right)=W
$$

so that $f_{2}$ is analytic in $S_{0}$ with

$$
\log ^{+}\left|f_{2}(z)\right|=O(\log |z|)
$$

Now (4.6) implies that

$$
W\left(f_{1}, f_{2}, f\right)=W F=W f / g^{2}
$$

so that, proceeding as in [2],

$$
W\left(w_{1}, w_{2}\right)=W
$$

where for $j=1,2$,

$$
w_{j}=f_{j}^{\prime} g+f_{j} h
$$

and

$$
h=-\left(f^{\prime} / f\right) g
$$

Now $g$ and $h$ are analytic in $S_{0}$, and therefore so is each $w_{j}$, so that (4.8) implies that $w_{1}, w_{2}$ are linearly independent solutions of an equation

$$
w^{\prime \prime}+a_{1} w^{\prime}+b_{1} w=0
$$


where $b_{1}$ is analytic in $S_{0}$. Here we used the fact that $W$ is non-vanishing in $S_{0}$. We claim that $b_{1}$ is in fact analytic in $|z|>R$, with at most a pole at infinity.

To establish this claim, first note that

$$
w_{1}=g f_{1}\left(\left(f_{1}^{\prime} / f_{1}\right)-\left(f^{\prime} / f\right)\right)
$$

so that $w_{1}^{\prime} / w_{1}$ is meromorphic in $|z|>R$, and therefore so is $b_{1}$. Now by Lemma 2 there is a sequence $r_{m} \rightarrow+\infty$ such that

$$
T\left(r_{m}, f^{\prime} / f\right)=O\left(r_{m}^{M_{1}}\right)
$$

for some positive $M_{1}$, so that writing (4.12) in the form $w_{1}=g f_{1} \phi$, and using (4.3) we obtain

$$
T\left(s_{m}, g^{2}\right)+T\left(s_{m}, \phi\right)=O\left(s_{m}^{M_{2}}\right)
$$

for some sequence $s_{m} \rightarrow+\infty$. Now (4.12), (4.13) and the lemma of the logarithmic derivative imply that

$$
m\left(r, b_{1}\right)=O(\log r)
$$

through a sequence of $r$ tending to infinity. To complete the proof of the claim, we note that we are free to define, in a sector $S_{1}=S(R, \alpha-\pi, \alpha+\pi)$, functions $g^{*}, h^{*}, W^{*}, f_{j}^{*}$ and $w_{j}^{*}$ in exactly the same way as $g, h, W, f_{j}$ and $w_{j}$ were defined above. Thus $w_{1}^{*}$ and $w_{2}^{*}$ are analytic solutions of some equation

$$
w^{\prime \prime}+a_{1} w^{\prime}+b_{1}^{*} w=0
$$

where $b_{1}^{*}$ is analytic in $S_{1}$. But it is quite clear that in the intersection of $S_{0}$ and $S_{1}, w_{1}^{*}$ and $w_{2}^{*}$ are linear combinations of $w_{1}$ and $w_{2}$, so that $b_{1}^{*}=b_{1}$ and $b_{1}$ is analytic in $S_{1}$. Now (4.14) implies that $b_{1}$ has at most a pole at infinity.

Suppose now that $b_{1}$ satisfies $b_{1}(z)=O\left(|z|^{-2}\right)$ as $z \rightarrow \infty$. Then the equation (4.11) has a regular singular point at infinity so that

$$
\log ^{+}\left|w_{j}(z)\right|=O(\log |z|)
$$

as $z \rightarrow \infty$ in $S_{0}$. But

$$
g=\left(w_{2} f_{1}-w_{1} f_{2}\right) / W
$$

so that using (4.7) and that fact that $W(z) z^{-\gamma_{2}}$ has a removable singularity at infinity we obtain

$$
\log ^{+}\left|g^{2}(z)\right|=O(\log |z|)
$$


in $S_{0}$. A similar estimate holds in $S_{1}$, so that $T(r, f / F)=O(\log r)$ and the conclusion of Theorem 1 now follows from Lemma 2.

We consider now the case where $b_{1}(z) \neq O\left(|z|^{-2}\right)$ as $z \rightarrow \infty$, and may write

$$
b_{1}(z)=\sum_{k=-\infty}^{n} a_{k}^{*} z^{k}
$$

in $|z|>R$ where $a_{n}^{*} \neq 0$ and $n \geqq-1$, and we set $N=(n+2) / 2 \geqq 1 / 2$. We shall eventually show that this case is impossible. We assert first that

$$
T(r, f / F)=O\left(r^{N}\right)
$$

For the region $|z|>R$ may be divided up into overlapping sectors in which using the method described in Section 3 we have

$$
\log ^{+}|w(z)|=O\left(|z|^{N}\right)
$$

for any solution of (4.11). Defining $g, h, W, f_{j}$ and $w_{j}$ in such sectors as above, and applying (4.15) we obtain (4.17). We now choose a real $\theta_{0}$ satisfying

$$
\operatorname{Arg}\left(a_{n}^{*}\right)+(n+2) \theta_{0}=0(\bmod 2 \pi)
$$

and take a small positive $\delta_{1}$. We take analytic solutions $f_{1}$ and $f_{2}$ of (4.4) in a sector $S_{2}=S\left(R_{1}, \theta_{0}-\delta_{1}, \theta_{0}+\delta_{1}\right)$ such that $W\left(f_{1}, f_{2}\right)=W$, and functions $g, h, w_{1}, w_{2}$ as in (4.3), (4.9) and (4.10), all defined exactly as in $S_{1}$, keeping the same notation for convenience. We define additional analytic functions $U_{1}, U_{2}, G_{1}$ and $G_{2}$ in $S_{2}$ by

$$
U_{j}=W^{-1 / 2} w_{j} \text { and } G_{j}=W^{-1 / 2} f_{j}
$$

for $j=1,2$. We have, by (4.6) and (4.8),

$$
W\left(U_{1}, U_{2}\right)=W\left(G_{1}, G_{2}\right)=1
$$

and $G_{1}, G_{2}$ solve an equation

$$
y^{\prime \prime}+A^{*} y=0
$$

where $A^{*}$ is analytic in $|z|>R$ with

$$
A^{*}(z)=O\left(|z|^{-2}\right)
$$

using (4.5). Also $U_{1}, U_{2}$ solve 


$$
u^{\prime \prime}+B u=0
$$

where

$$
B(z)=a_{n}^{*} z^{n}(1+o(1))
$$

is analytic in $|z|>R$. Now provided $R_{1}$ is sufficiently large and $\delta_{1}$ is sufficiently small, the equation (4.23) has in $S_{2}$ linearly independent solutions $u_{1}, u_{2}$ given by

$$
u_{k}(z)=B(z)^{-1 / 4} v_{k}(z)=B(z)^{-1 / 4}(1+o(1)) \exp \left((-1)^{k+1} i Z\right)
$$

where for a suitable point $z_{0}$,

$$
Z=\int_{z_{0}}^{z} B(t)^{1 / 2} d t=-i \beta z^{N}+O\left(|z|^{N-1 / 2}\right)
$$

Here $\beta$ is a non-zero constant, and by (4.18), $\operatorname{Re}\left(\beta z^{N}\right)=0$ on $\arg z=\theta_{0}$. We can write

$$
U_{k}(z)=B(z)^{-1 / 4}\left(C_{k} v_{1}+D_{k} v_{2}\right)
$$

for constants $C_{1}, C_{2}, D_{1}$ and $D_{2}$, where $C_{1}$ and $C_{2}$ cannot both vanish, and nor can $D_{1}$ and $D_{2}$. Finally we set

$$
\phi_{1}=C_{2} G_{1}-C_{1} G_{2} \text { and } \phi_{2}=D_{2} G_{1}-D_{1} G_{2}
$$

and note that by (4.15), (4.19) and (4.27),

$$
g=u_{1} \phi_{1}+u_{2} \phi_{2}
$$

We make some observations about $G_{1}$ and $G_{2}$. Now $G_{1}$ is determined from $f_{1}$, which is chosen so that $f_{1} z^{-y_{1}}$ has a removable singularity at infinity. Further, $G_{2}$ is determined from $f_{2}$, which is required only to satisfy $W\left(f_{1}, f_{2}\right)=W$. Thus we are free to choose $G_{2}$ subject only to (4.20). We can assume therefore that in $S_{2}$,

$$
G_{1}(z)=z^{v}(1+O(1 /|z|))
$$

and that since

$$
\left(G_{2} / G_{1}\right)^{\prime}=G_{1}^{-2}=z^{-2 v}(1+O(1 /|z|)),
$$

we have

$$
G_{2} / G_{1}=(1-2 v)^{-1} z^{1-2 v}(1+o(1))
$$


or, if $v=1 / 2$,

$$
G_{2} / G_{1}=\log z+o(1)
$$

in $S_{2}$. We thus have

$$
G_{1} G_{2}=z(1-2 v)^{-1}(1+o(1)) \quad \text { or } \quad G_{1} G_{2}=(z \log z)(1+o(1))
$$

in $S_{2}$. We shall establish the following Claim:

Claim. There exist constants $\lambda>1$ and $K>0$ with the following property. There exist arbitrarily large positive $r$ such that in

$$
\lambda^{-1} r<|z|<\lambda r, \quad\left|\arg z-\theta_{0}\right|<\delta_{1},
$$

$f(z)$ has no poles, while for $j=1,2$,

$$
|\log | \phi_{j}(z)|| \leqq K \log |z| .
$$

To prove this Claim we set

$$
H=\left(U_{1} / U_{2}\right)\left(f_{2} / f_{1}\right)=\left(U_{1} / U_{2}\right)\left(G_{2} / G_{1}\right)
$$

By (4.9) and (4.19),

$$
f^{\prime} / f=(1-H)^{-1}\left(\left(f_{1}^{\prime} / f_{1}\right)-\left(H f_{2}^{\prime} / f_{2}\right)\right),
$$

and thus at a large pole of $f$ in $S_{2}$ we must have $H=1$, since $f_{1}$ and $f_{2}$ are non-zero for large $z$ in $S_{2}$, by (4.30) and (4.31) or (4.32). Also a pole of $f$ is a zero of $g$, but not of $h$, so that $U_{1}$ and $U_{2}$ cannot vanish at a large pole of $f$, and $H$ cannot have pole there. Also

$$
\left(f_{1}^{\prime} / f_{1}\right)-\left(f_{2}^{\prime} / f_{2}\right)=-W /\left(f_{1} f_{2}\right)=-1 /\left(G_{1} G_{2}\right)
$$

Thus at a large pole of $f$ in $S_{2}$, we have $H=1$ and $f^{\prime} / f$ has a simple pole with residue equal to

$$
\left(-1 /\left(G_{1} G_{2}\right)\right) /\left(-H^{\prime}\right)=\left(\left(H^{\prime} / H\right)\left(G_{1} G_{2}\right)\right)^{-1}=\left(1-\left(G_{1} G_{2}\right) /\left(U_{1} U_{2}\right)\right)^{-1}
$$

by (4.20) and (4.35).

Assume for the time being that $\operatorname{Re}(1-2 v) \neq 0$ and that $G_{1} / G_{2} \rightarrow 0$ in $S_{2}$, that is that $\operatorname{Re}(1-2 v)>0$. At a large pole of $f$ in $S_{2}, H=1$ gives

$$
G_{1} / G_{2}=\left(C_{1} v_{1}+D_{1} v_{2}\right) /\left(C_{2} v_{1}+D_{2} v_{2}\right)
$$


using (4.27). We note that by (4.25),

$$
v_{1} v_{2} \rightarrow 1
$$

as $z \rightarrow \infty$ in $S_{2}$. Recalling that $U_{1}, U_{2}$ are non-zero at a large pole $\zeta$ of $f$ in $S_{2},(4.37)$ gives

$$
\left(C_{1}+o(1)\right) v_{1}(\zeta)+\left(D_{1}+o(1)\right) v_{2}(\zeta)=0 .
$$

If $C_{1}=0$, then $D_{1} \neq 0$ and (4.38) and (4.39) yield

$$
\left(C_{1} v_{1}+D_{1} v_{2}\right)\left(C_{2} v_{1}+D_{2} v_{2}\right)=O(1)
$$

at $\zeta$. The same conclusion (4.40) holds if $D_{1}=0$ and if $C_{1} D_{1} \neq 0$. Now (4.40) implies that

$$
U_{1}(\zeta) U_{2}(\zeta)=O\left(|B(\zeta)|^{-1 / 2}\right)=O\left(|\zeta|^{1 / 2}\right),
$$

using (4.27). Now (4.33) and (4.41) yield

$$
\left(U_{1}(\zeta) U_{2}(\zeta)\right) /\left(G_{1}(\zeta) G_{2}(\zeta)\right)=O\left(|\zeta|^{-1 / 2}\right)
$$

which on substitution into (4.36) gives a contradiction, since the residue (4.36) is required to be a negative integer. In this case therefore $f$ can have only finitely many poles in $S_{2}$. By the obvious symmetry of (4.37) and the fact that we are only concerned with the products $G_{1} G_{2}$ and $U_{1} U_{2}$ in (4.36), the same conclusion holds if $\operatorname{Re}(1-2 v)<0$ so that $G_{2} / G_{1} \rightarrow 0$ in $S_{2}$.

Now suppose that $1-2 v=0$. In this case, by (4.32), $G_{1} / G_{2} \rightarrow 0$ in $S_{2}$ again, so that as above we obtain (4.39) and (4.40) at a large pole $\zeta$ of $f$ in $S_{2}$. By (4.33), (4.42) now becomes

$$
\left(U_{1}(\zeta) U_{2}(\zeta)\right) /\left(G_{1}(\zeta) G_{2}(\zeta)\right)=O\left(\left(|\zeta|^{1 / 2} \log |\zeta|\right)^{-1}\right)
$$

and we have a contradiction as before.

We have thus proved that if $\operatorname{Re}(1-2 v) \neq 0$ or if $v=1 / 2$ then $f$ has only finitely many poles in $S_{2}$. Moreover, in this case (4.34) holds for all sufficiently large $z$ in $S_{2}$, by (4.30) and (4.31) or (4.32).

We still need to establish the Claim in the case where $1-2 v=i \mu$, where $\mu$ is real and non-zero. In this case, $\log \left|G_{1} / G_{2}\right|=O(1)$ in $S_{2}$. For a large pole $\zeta$ of $f$ in $S_{2}$ we again have (4.37), which gives, at $\zeta$,

$$
\left(C_{1}-C_{2} G_{1} / G_{2}\right) v_{1}+\left(D_{1}-D_{2} G_{1} / G_{2}\right) v_{2}=0 .
$$

Using (4.38), this equation clearly gives 


$$
\left|v_{1}(\zeta)\right|+\left|v_{2}(\zeta)\right|=O(1)
$$

unless one of the coefficients of $v_{1}, v_{2}$ in (4.43) is small. Now (4.44) leads to (4.41) again, and we obtain (4.42) and a contradiction as before. Therefore in this case, for any $\varepsilon_{1}>0$, $f$ has only finitely many poles in $S_{2}$ outside the set where

$$
\left|C_{1}-C_{2} G_{1} / G_{2}\right|<\varepsilon_{1} \text { or }\left|D_{1}-D_{2} G_{1} / G_{2}\right|<\varepsilon_{1} \text {. }
$$

By (4.31) it is clear that there are regions free of such points as described in the Claim. In such regions (4.34) follows from (4.30) and (4.31), and the Claim is proved in all cases.

We are now in a position to obtain a contradiction, and show that the case $b_{1}(z) \neq O\left(|z|^{-2}\right)$ is impossible. We take a region

$$
\lambda^{-1} r<|z|<\lambda r, \quad\left|\arg z-\theta_{0}\right|<\delta_{1},
$$

with $r$ large, on which $f$ has no poles and hence $g$ has no zeros, and on which (4.34) holds. Setting $\rho=\lambda^{1 / 2}$, standard estimates yield a positive constant $K_{2}$ such that

$$
\left|g^{\prime}(z) / g(z)\right| \leqq K_{2} r^{N-1}
$$

on

$$
\rho^{-1} r \leqq|z| \leqq \rho r, \quad\left|\arg z-\theta_{0}\right| \leqq \delta_{1} / 2
$$

For $g^{2}$ is analytic on $|z| \geqq R$, and by (4.17) can be represented as the product of a power of $z$, a function with a removable singularity at infinity, and an entire function $h_{3}$ with $T\left(s, h_{3}\right)=O\left(s^{N}\right)$. We recall that $g=u_{1} \phi_{1}+u_{2} \phi_{2}$, and that $u_{1}$ and $u_{2}$ are given by (4.25) and (4.26), where $\operatorname{Re}\left(\beta z^{N}\right)=0$ on $\arg z=\theta_{0}$. We assume without loss of generality that $\operatorname{Re}\left(\beta z^{N}\right)>0$ on $\theta_{0}<\arg z<\theta_{0}+\delta_{1}$. For any fixed $\delta_{2}$ in $\left(0, \delta_{1} / 2\right)$, we have, if $r$ is large enough,

$$
\left|\log g(z)-\log u_{k}(z)\right| \leqq K_{3} \log |z|
$$

on $\rho^{-1} r \leqq|z| \leqq \rho r, \arg z=\theta_{0}+(-1)^{k+1} \delta_{2}$. Here $K_{3}$ is a positive constant, and (4.46) follows from (4.25) and (4.34). Let $\Gamma$ be the contour consisting of the arcs

$$
|z|=\rho^{-1} r, \quad|z|=\rho r, \quad\left|\arg z-\theta_{0}\right| \leqq \delta_{2},
$$

and the straight line segments joining their end-points, and let the vertices of $\Gamma$ be $X_{1}$, $Y_{1}, Y_{2}, X_{2}$, ordered anti-clockwise, with $X_{1}=\rho^{-1} r \exp \left(i\left(\theta_{0}-\delta_{2}\right)\right)$. By (4.45) there is a positive constant $K_{4}$ such that the integral of $g^{\prime} / g$ around the curved parts of $\Gamma$, described in the positive sense, has modulus at most $\delta_{2} K_{4} r^{N}$. Choose $\delta_{2}$ so small that 


$$
\left|\delta_{2} K_{4}\right|<\left|\beta e^{i N \theta_{0}}\right|\left(\rho^{N}-\rho^{-N}\right) \cos \left(N \delta_{2}\right)
$$

By (4.25), (4.26) and (4.46), if $r$ is large enough,

$$
\log g\left(Y_{1}\right)-\log g\left(X_{1}\right)+\log g\left(X_{2}\right)-\log g\left(Y_{2}\right)=-2 \beta e^{i N \theta_{0}} r^{N}\left(\rho^{N}-\rho^{-N}\right) \cos \left(N \delta_{2}\right)+\eta
$$

where, for some positive constant $K_{5}$,

$$
|\eta|<K_{5}\left(r^{N-1 / 2}+\log r\right)
$$

If $r$ is large enough, this contradicts the argument principle.

\section{Proof of Theorem 2}

Suppose that $f(z)$ is meromorphic in the plane and that $f$ and $F$ have only finitely many zeros, where

$$
F(z)=f^{\prime \prime}(z)-\alpha f(z)
$$

and $\alpha$ is a non-zero constant. Suppose further that $\bar{N}(r, f)$ has finite lower order. By results from [2], where we apply Theorem 1 if $f$ is entire and Lemma 8 if $f$ is not entire, $f^{\prime} / f$ has finite lower order. We shall prove that either $f$ is given by (1.2), or $f$ has finite order, so that in the latter case we can appeal to Theorem C. Clearly we may assume that $\alpha=1$, that $f$ is transcendental, and that $F$ is non-constant.

We begin by defining a rational function $R$ and an entire function $g$ by

$$
g(z)^{2}=R(z) f(z) / F(z)
$$

Now $g$ has finite lower order, and so has

$$
h(z)=-\left(f^{\prime}(z) / f(z)\right) g(z)
$$

which has only finitely many poles. Indeed there is a sequence $s_{n}$ tending to infinity such that

$$
T\left(s_{n}, g\right)+T\left(s_{n}, h\right)=O\left(s_{n}^{M_{1}}\right)
$$

for some positive $M_{1}$. To see this, we need only take a corresponding sequence $s_{n}^{\prime}$ for $T\left(r, f^{\prime} / f\right)$ and if necessary make $s_{n}$ slightly smaller. Now

$$
W\left(e^{z}, e^{-z}, f\right)=-2 F=-2 R f / g^{2}
$$

so that

$$
W\left(w_{1}, w_{2}\right)=-2 R
$$

where for $j=1,2$,

$$
w_{j}=f_{j} h+f_{j}^{\prime} g
$$


with

$$
f_{1}(z)=e^{z}, \quad f_{2}(z)=e^{-z}
$$

Each $w_{j}$ is meromorphic in the plane with only finitely many poles and with finite lower order, by (5.4). Now (5.5) implies that $w_{1}, w_{2}$ are linearly independent solutions of an equation

$$
w^{\prime \prime}-\left(R^{\prime} / R\right) w^{\prime}+b_{1} w=0
$$

where $b_{1}(z)$ is meromorphic in the plane and has only finitely many poles, since poles of $b_{1}$ can only arise from poles of the $w_{j}$ and zeros of their Wronskian. Since $w_{1}$ has finite lower order the lemma of the logarithmic derivative implies that $b_{1}(z)$ is rational. We shall show that $b_{1}(\infty) \neq 0, \infty$ by considering the two contrary cases $b_{1}(\infty)=0, b_{1}(\infty)=\infty$ separately.

Suppose that $b_{1}(\infty)=0$. Then by Lemma 1 we must have $b_{1}(z)=O\left(|z|^{-2}\right)$ as $z \rightarrow \infty$, so that (5.8) has a regular singular point at infinity. Thus each $w_{j}$ is rational, and by (5.6) and (5.7) we obtain

$$
f^{\prime} / f=\left(v_{1}+1\right) /\left(v_{1}-1\right)
$$

where $v_{1}=T_{1} e^{2 z}$ and $T_{1}$ is rational. Thus $f^{\prime} / f$ has infinitely many poles at points where $v_{1}=1$, and at a large such pole $f^{\prime} / f$ has residue

$$
2 /\left(v_{1}^{\prime}\right)=2 v_{1} / v_{1}^{\prime}=1+o(1) .
$$

But this implies that $f$ has infinitely many zeros, which is a contradiction.

Suppose that $b_{1}(\infty)=\infty$. Then we can write

$$
b_{1}(z)=a_{n} z^{n}+a_{n-1} z^{n-1}+\cdots
$$

for large $z$, where $n$ is at least 1 and $a_{n} \neq 0$. Set $N=(n+2) / 2$. We observe first that the plane can be divided up into overlapping sectors in which we have (see Section 3 )

$$
\log ^{+}|w(z)|=O\left(|z|^{N}\right)
$$

for every solution of (5.8). Solving (5.6) for $g$ we obtain a similar estimate for $\log ^{+}|g(z)|$ and deduce that

$$
T(r, g)=O\left(r^{N}\right)
$$

We now take $\theta_{0}$ in $(-\pi / 2, \pi / 2)$ satisfying 


$$
\operatorname{Arg}\left(a_{n}\right)+(n+2) \theta_{0}=0(\bmod 2 \pi)
$$

and will proceed to a contradiction. We shall first establish the following Claim.

Claim 1. For any positive $\varepsilon_{1}$ and $r_{1}, f$ has infinitely many poles in the sector $S_{1}=S\left(r_{1}, \theta_{0}-\varepsilon_{1}, \theta_{0}+\varepsilon_{1}\right)$

We again use the method of asymptotic integration as described in Section 3, and a fairly standard application of the argument principle. Obviously we may assume that $r_{1}$ is large and that $\varepsilon_{1}$ is small. Now the equation (5.8) has linearly independent solutions $u_{1}$ and $u_{2}$ in $S_{1}$ given by

$$
u_{j}(z)=R(z)^{1 / 2} b(z)^{-1 / 4}(1+o(1)) \exp \left((-1)^{j+1} i Z\right)
$$

where

$$
b(z)=b_{1}(z)+R^{\prime \prime} / 2 R-3 R^{\prime 2} / 4 R^{2}
$$

and

$$
i Z=\beta_{1} z^{N}+O\left(|z|^{N-1 / 2}\right)
$$

Here $\beta_{1}$ is a non-zero constant. Also $\operatorname{Re}\left(\beta_{1} z^{N}\right)=0$ on $\arg z=\theta_{0}$, and we may assume that $\operatorname{Re}\left(\beta_{1} z^{N}\right)>0$ on $S\left(r_{1}, \theta_{0}, \theta_{0}+\varepsilon_{1}\right)$. Now we can write

$$
u_{j}=F_{j}^{\prime} g+F_{j} h
$$

for $j=1,2$, where $F_{1}, F_{2}$ are linearly independent solutions of $y^{\prime \prime}-y=0$, and so

$$
g=\left(u_{1} F_{2}-u_{2} F_{1}\right) / W\left(F_{2}, F_{1}\right)
$$

Now (5.12), (5.13), (5.14) and obvious estimates for $F_{1}, F_{2}$ imply that for any sufficiently small positive $\delta_{1}$ there exists $r_{2}>0$ such that for $|z| \geqq r_{2}, \arg z=\theta_{0}+(-1)^{j} \delta_{1}$, we have

$$
\log g(z)=(-1)^{j} \beta_{1} z^{N}+O\left(|z|^{N-1 / 2}\right)
$$

Here we have used the fact that $F_{1}$ and $F_{2}$ cannot vanish in $S_{1}$, by the choice of $\theta_{0}$. Now if $f$ has only finitely many poles in $S_{1},(5.10)$ and routine estimates yield a positive constant $K_{1}$ such that

$$
\left|g^{\prime}(z) / g(z)\right| \leqq K_{1}|z|^{N-1}
$$

in $S\left(r_{3}, \theta_{0}-\varepsilon_{1} / 2, \theta_{0}+\varepsilon_{1} / 2\right)$ if $r_{3}$ is large. Take a large $r_{4}$ and $r_{5}$ with $r_{5} / r_{4}$ large, and let $\Gamma$ 
be the contour consisting of the circular arcs from $r_{k} \exp \left(i\left(\theta_{0}+\delta_{1}\right)\right)$ to $r_{k} \exp \left(i\left(\theta_{0}-\delta_{1}\right)\right)$, for $k=4,5$, and the straight line segments joining them, $\Gamma$ described anti-clockwise. Now the integral of $g^{\prime} / g$ around the two circular arcs in the positive sense has, by (5.16), modulus at most $K_{2} \delta_{1} r_{5}^{N}$ for some positive constant $K_{2}$. Choose $\delta_{1}$ so small that $K_{2} \delta_{1}<\left|\beta_{1} e^{i N \theta_{0}} \cos N \delta_{1}\right|$. By (5.15), if $r_{4}$ is large enough, then the integral of $g^{\prime} / g$ along the two straight line segments, again in the positive sense, is equal to

$$
-2 \beta_{1}\left(r_{5}^{N}-r_{4}^{N}\right) e^{i N \theta_{0}} \cos N \delta_{1}+O\left(r_{5}^{N-1 / 2}\right) .
$$

This is clearly impossible, and Claim 1 is proved.

We now examine the residues of the poles of $f^{\prime} / f$ in $S_{1}$, to obtain a contradiction and thus show that $b_{1}(\infty)=\infty$ is impossible. By the choice of $\theta_{0}$, we may assume that

$$
\left|e^{2 z}\right|>|z|^{2 n}
$$

in $S_{1}$. Now (5.6) yields

$$
f^{\prime} / f=(1+H) /(1-H)
$$

where

$$
H=e^{-2 z} w_{1} / w_{2}
$$

At a pole of $f$ in $S_{1}$ we have $H=1$, and $f^{\prime} / f$ has a simple pole with residue $-2 / H^{\prime}$. At a 1-point of $H$,

$$
H^{\prime}=H^{\prime} / H=-2+W\left(w_{2}, w_{1}\right) /\left(w_{1} w_{2}\right)=-2+2 R /\left(w_{1} w_{2}\right)
$$

using (5.5). So the multiplicity of a large pole of $f$ in $S_{1}$ is

$$
\left(-1+R /\left(w_{1} w_{2}\right)\right)^{-1} \text {. }
$$

Set

$$
W_{j}(z)=R(z)^{-1 / 2} b(z)^{1 / 4} w_{j}(z)
$$

At a large pole of $f$ in $S_{1}, \zeta$ say, we have

$$
e^{2 \zeta}=W_{1}(\zeta) / W_{2}(\zeta)
$$

We may write, by (5.12),

$$
W_{j}(z)=C_{j}(1+o(1)) e^{i Z}+D_{j}(1+o(1)) e^{-i Z}
$$

in $S_{1}$, for some constants $C_{1}, D_{1}, C_{2}, D_{2}$, and observe that there are positive constants 
$\varepsilon_{2}$ and $K_{3}$ such that if $\zeta$ is large and $\left|W_{2}(\zeta)\right|<\varepsilon_{2}$ then $\left|W_{1}(\zeta) W_{2}(\zeta)\right| \leqq K_{3}$. But this last estimate gives, using (5.21),

$$
\left|w_{1}(\zeta) w_{2}(\zeta)\right|=O\left(|R(\zeta)||b(\zeta)|^{-1 / 2}\right)=O\left(|R(\zeta)||\zeta|^{-1 / 2}\right)
$$

which on substitution into (5.20) yields a contradiction. On the other hand, $\left|W_{2}(\zeta)\right| \geqq \varepsilon_{2}$ leads to

$$
\left|W_{2}(\zeta) W_{1}(\zeta)\right| \geqq|\zeta|^{2 n} \varepsilon_{2}^{2}
$$

by (5.17) and (5.22), so that in this case

$$
\left|w_{2}(\zeta) w_{1}(\zeta)\right| \geqq|R(\zeta)||\zeta|^{n}
$$

if $\zeta$ is large enough, and again (5.20) gives a contradiction.

We are therefore left only to consider the case where $b_{1}(\infty) \neq 0, \infty$, the alternative cases having each led to a contradiction. Now by Lemma 1, the equation (5.8) has linearly independent solutions $V_{1} e^{A z}, V_{2} e^{-A z}$, where $V_{1}$ and $V_{2}$ are rational, and $A$ is a non-zero constant. Now (5.3), (5.6) and (5.7) give

$$
\frac{f^{\prime}-f}{f^{\prime}+f}=e^{-2 z} \frac{A_{1} V_{1} e^{A z}+B_{1} V_{2} e^{-A z}}{A_{2} V_{1} e^{A z}+B_{2} V_{2} e^{-A z}}
$$

where $A_{1}, A_{2}, B_{1}, B_{2}$ are constants. We note that $A_{1}$ and $A_{2}$ cannot both vanish, and nor can $B_{1}$ and $B_{2}$, since $w_{1}$ and $w_{2}$ are linearly independent. We shall complete the proof of Theorem 2 by dividing this case up into subcases.

Subcase 1. Suppose that $A_{1}=B_{2}=0$, or $B_{1}=A_{2}=0$.

Then (5.23) yields

$$
f^{\prime} / f=-1+2 /\left(1-v_{2}\right)
$$

where $v_{2}=V_{3} e^{d_{1} z}$ with $V_{3}$ rational, and $d_{1}$ a constant. If $d_{1}=0$, then obviously $f^{\prime} / f$ is rational. If $d_{1} \neq 0$ then $f^{\prime} / f$ has infinitely many poles at points where $v_{2}=1$, with residue

$$
-2 / v_{2}^{\prime}=-2 /\left(\left(V_{3}^{\prime}+d_{1} V_{3}\right) e^{d_{1} z}\right)=-2 / d_{1}+o(1)
$$


So $2 / d_{1}$ must be a positive integer, and $V_{3}$ must in fact be constant, and $f$ is given by (1.3).

Subcase 2. Suppose that $A_{1} B_{1} A_{2} B_{2} \neq 0$.

We shall show that in this case $f$ has finite order. From (5.23) we see that there exist a real $\theta_{1}$ and positive $r_{6}, K_{4}$ and $K_{5}$ such that for large $z$ outside the semi-infinite "logarithmic" strips

$$
\Omega_{j}=\left\{z:|z|>r_{6},\left|\arg z+(-1)^{j} \theta_{1}\right|<K_{4} \log |z| /|z|\right\}
$$

we have

$$
\left(f^{\prime}-f\right) /\left(f^{\prime}+f\right)=e^{-2 z} \phi(z)
$$

where $|\log | \phi(z) \| \leqq K_{5}$. This clearly gives

$$
f^{\prime}(z) / f(z)=O(1)
$$

for large $z$ outside $\Omega_{1}, \Omega_{2}$ and the semi-infinite strips $\Omega_{3}, \Omega_{4}$ given by $|z|>r_{6}$, $|\operatorname{Re}(z)|<K_{6}$, say. For a large $z$ outside the $\Omega_{j}$ this leads to

$$
\log ^{+}|1 / f(z)|=O(|z|)
$$

Now setting $G=f^{\prime} / f,(5.23)$ yields $T(r, G)=O(r)$. By Theorem 3 of [5], we can certainly obtain

$$
\left|G^{\prime}(z) / G(z)\right| \leqq(\log r)^{4}
$$

for all $z$ on $|z|=r$, where $r$ lies outside a set of finite logarithmic measure. For such $r$, (5.25) and (5.27) lead to

$$
G(z)=O\left(\exp (\log r)^{Q}\right)
$$

on $|z|=r$, for some positive $Q$, and hence using (5.26) we obtain

$$
\log ^{+}|1 / f(z)|=O\left(\exp (\log r)^{2 Q}\right)
$$

on the same circle, if $r$ is large. On the other hand, if $\Omega$ is one of $\Omega_{1}, \Omega_{2}, \Omega_{3}, \Omega_{4}$, and $A_{3}, A_{4}$ are suitably chosen constants, then by (5.26) the subharmonic function

$$
\psi(z)=\log ^{+}\left|e^{A_{3} z+A_{4}} f(z)^{-1}\right|
$$

vanishes on the boundary of $\Omega$. Estimates for harmonic measure [4, p. 104] now show that either $\psi$ vanishes for all $z$ in $\Omega$, or 


$$
\liminf _{s \rightarrow \infty} m(s) \exp \left(-\pi \int_{r 6}^{s} d t / t \theta(t)\right)>0
$$

where $m(s)=\sup \{\psi(z):|z|=s, z$ in $\Omega\}$ and $\theta(t)$ is the angular measure of the intersection of $\Omega$ with $|z|=r$. But $\theta(t)=O((\log t) / t)$ so that (5.29) yields $\log m(s)>K_{7} s /(\log s)$, say, which contradicts (5.28). Thus $\psi(z) \equiv 0$ on $\Omega$ and (5.26) holds for all large $z$.

Subcase 3. Suppose that exactly one of $A_{1}, A_{2}, B_{1}, B_{2}$ is zero.

Then without loss of generality (5.23) gives

$$
\left(f^{\prime}-f\right) /\left(f^{\prime}+f\right)=A_{1} e^{-2 z}+B_{1} V e^{B z}
$$

or

$$
\left(f^{\prime}-f\right) /\left(f^{\prime}+f\right)=\left(A_{2} e^{2 z}+B_{2} V e^{B z}\right)^{-1}
$$

with $B$ constant and $V$ rational. We may assume that (5.30) holds for otherwise we need only set $f_{3}(z)=f(-z)$ and apply the following argument to $f_{3}$. Now (5.30) gives

$$
f^{\prime} / f=-1+2 /\left(1-A_{1} e^{-2 z}-B_{1} V e^{B z}\right) \text {. }
$$

If $B=-2$, then (5.32) shows that $f^{\prime} / f$ is bounded at least for large $z$ outside a pair of logarithmic strips, so that we can apply the argument of the previous section to conclude that $f$ has finite order. The same conclusion holds if $B \neq 0,-2$, except that we may need more than two, but at most six, logarithmic strips if $B_{1} V$ does not vanish identically. Finally if $B=0$, we obtain

$$
f^{\prime} / f=-1+2 /\left(V_{4}-A_{1} e^{-2 z}\right)
$$

with $V_{4}$ rational. In this case either $V_{4}$ is identically zero, in which case $f$ is given by (1.2) or $f^{\prime} / f$ has infinitely many poles at points where $V_{4}=A_{1} e^{-2 z}$, with residue $2 /\left(V_{4}^{\prime}+2 V_{4}\right)$. Thus a large pole $\zeta$ of $f$ has multiplicity bounded by a power of $|\zeta|$, so that $N(r, f)$ has finite order. We can thus write $f=e^{g_{0}} / H_{1}$, where $H_{1}$ has finite order and $g_{0}$ is entire. But Lemma 8 of [2] gives $m\left(r, f^{\prime} / f\right)=O(\log r)$, and $g_{0}$ is a polynomial. Now $f$ has finite order and Theorem 2 is proved.

\section{REFERENCES}

1. L. Bieberbach, Theorie de gewöhnlichen Differentialgleichungen, 2nd ed. (Springer Verlag, Berlin, 1965).

2. G. Frank and S. Hellerstein, On the meromorphic solutions of nonhomogeneous linear differential equations with polynomial coefficients, Proc. London Math. Soc. (3) 53 (1986), 407-428.

3. G. Frank, W. Hennekemper and G. Polloczek, Uber die Nullstellen meromorpher Funktionen und ihrer Ableitungen, Math. Ann. 225 (1977), 145-154. 
4. W. H. J. Fuchs, Topics in the Theory of Functions of One Complex Variable (Van Nostrand Math. Studies, 12, 1967).

5. G. Gundersen, Estimates for the logarithmic derivative of a meromorphic function, plus similar estimates, J. London Math. Soc. (2) 37 (1988), 88-104.

6. W. K. Hayman, Meromorphic Functions (Clarendon Press, Oxford, 1964).

7. S. Hellerstein and J. Rossi, Zeros of meromorphic solutions of second order linear differential equations, Math. Z. 192 (1986), 603-612.

8. E. Hille, Lectures on Ordinary Differential Equations (Addison-Wesley, Reading, Mass., 1969).

9. E. HiLle, Ordinary Differential Equations in the Complex Domain (Wiley, New York, 1976).

10. E. L. INCE, Ordinary Differential Equations (Dover, N.Y., 1956).

11. J. K. Langley, On the zeros of linear differential polynomials with small rational coefficients, J. London Math. Soc. (2) 36 (1987), 445-457.

12. J. K. Langley, On the zeros of $\left(f^{\prime \prime}+\alpha f\right) f$ and a result of Steinmetz, Proc. Roy. Soc. Edinburgh 108A (1988), 241-247.

13. J. K. Langley, The Tsuji characteristic and zeros of linear differential polynomials, Analysis, to appear.

14. E. Mues, Uber ein Vermutung von Hayman, Math. Z. 119 (1972), 11-20.

15. N. Steinmetz, On the zeros of $\left(f^{(p)}+a_{p-1} f^{(p-1)}+\cdots+a_{0} f\right) f$, Analysis 7 (1987), 375-389.

16. G. Valiron, Lectures on the General Theory of Integral Functions (Edouard Privat, Toulouse, 1923).

Department of Mathematics

UnIVERSITY COLlege LONDON

LONDON WC1E 6BT 